\title{
Punicalagin inhibited proliferation, invasion and angiogenesis of osteosarcoma through suppression of NF- $\mathrm{NB}$ signaling
}

\author{
TAO HUANG, XIN ZHANG and HAIPENG WANG \\ Department of Orthopedic Surgery, The Second Affiliated Hospital of Air Force Medical University, \\ Xi'an, Shaanxi 710038, P.R. China
}

Received October 2, 2019; Accepted May 12, 2020

DOI: $10.3892 / \mathrm{mmr} .2020 .11304$

\begin{abstract}
Osteosarcoma is the most common primary malignant bone tumor among children and young people and is associated with poor prognosis. Punicalagin is an antioxidant ellagitannin found in pomegranate juice with known antiproliferation and anti-angiogenesis properties. However, the antitumor effect of punicalagin on osteosarcoma requires further investigation. In the present study, the inhibitory effect of punicalagin on proliferation and invasion was evaluated in one human osteoblast cell line (hFOB1.19) and three human osteosarcoma cell lines (U2OS, MG63 and SaOS2). The cancer cell apoptosis ratio was determined using flow cytometry. NF- $\kappa \mathrm{B}$ signaling in these cells was also evaluated using western blotting analysis. A subcutaneous tumor xenograft model was initiated to study the efficacy of punicalagin on osteosarcoma development and angiogenesis in vivo. Punicalagin treatment significantly decreased osteosarcoma cell proliferation and increased apoptosis. In addition, the invasion potential of these cells in a transwell assay was also dramatically suppressed in osteosarcoma cells. Punicalagin not only induced the degradation of I $\mathrm{B} \alpha$ but also the nuclear translocation of p65, suggesting the attenuation of NF- $\kappa \mathrm{B}$ signaling pathway following treatment. Moreover, punicalagin markedly downregulated interleukin (IL)-6 and IL-8 levels, which was consistent with the inhibition of NF- $\mathrm{BB}$ signaling. An NF- $\mathrm{B}$ activator could reverse these effects. Using a tumor xenograft mouse model, it was demonstrated that punicalagin exposure inhibited osteosarcoma growth and angiogenesis in vivo. These observations confirmed the suppressive effect of punicalagin against osteosarcoma malignancies. The underlying molecular mechanisms may include inhibition of the NF- $\mathrm{KB}$ signaling pathway.
\end{abstract}

Correspondence to: Dr Haipeng Wang, Department of Orthopedic Surgery, The Second Affiliated Hospital of Air Force Medical University, 569 Xinsi Road, Baqiao, Xi'an, Shaanxi 710038, P.R. China E-mail: haipeng_wangcn@sina.com

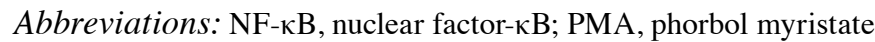
acetate; mTOR, mammalian target of rapamycin; Ctrl, control

Key words: osteosarcomas, punicalagin, $\mathrm{NF}-\kappa \mathrm{B}$ pathway, malignancy

\section{Introduction}

Osteosarcoma accounts $<1 \%$ of malignancies overall, with an incidence of $\sim 5$ cases per million in individuals $<19$ years of age in the USA. However, it is the most widely diagnosed primary malignant bone tumor, particularly among children and young people. Males are prone to the onset of osteosarcoma, and the ratio of male to female incidence is $\sim 3: 2$ (1). Osteosarcoma is thought to arise from mesenchymal primitive bone-forming cells, and is characterized by the sustainable production of malignant osteogenesis. In addition, the production of pro-angiogenic factors in the malignant development of osteosarcoma has also been suggested by a previous study, which concluded that osteosarcoma had a strong tendency to metastasize early and was associated with poor prognosis (2). Thus, the degree of osteosarcoma malignancy is extremely high, and increased tumor invasiveness and vascularity are associated with metastatic potential and poor prognosis.

The main current treatment strategy for patients with newly diagnosed osteosarcoma includes neoadjuvant chemotherapy, followed by surgical removal of the primary tumor and all metastatic lesions with clinical manifestations, as well as postoperative adjuvant chemotherapy (3). The three-drug chemotherapy regimen of cisplatin, doxorubicin and methotrexate constitutes the primary option for backbone treatment, and the overall 5-year survival rate in America for osteosarcoma has increased to $60-70 \%$ in patients receiving the three-drug regimen (4). Recently, biologic agents, such as muramyl tripeptide and IFN- $\alpha-2 b$, and additional cytotoxic chemotherapy, such as ifosfamide, have been introduced into clinical trials. However, these have failed to significantly improve the survival of young patients with osteosarcoma $(5,6)$. Therefore, an improved understanding of the underlying mechanisms of tumor progression and angiogenesis in osteosarcoma is required in order to identify and develop more effective therapies.

Dysregulation in nuclear factor- $\kappa \mathrm{B}(\mathrm{NF}-\kappa \mathrm{B})$ signaling is associated with excessive cellular proliferation and developmental signals during tumorigenesis. Indeed, this pathway has been reported to be involved in inflammatory proliferation and differentiation of osteosarcoma cells (7). $\mathrm{NF}-\kappa \mathrm{B}$ can also regulate the generation of proinflammatory and proangiogenic cytokines around cancer cells (8). Thus, it 
was previously suggested that $\mathrm{NF}-\kappa \mathrm{B}$ could serve a causative role in osteosarcoma progression (9). Punicalagin is an antioxidant ellagitannin found in pomegranate juice with known anti-proliferation or anti-angiogenesis properties against many cancer cell lines, including leukemia, glioma and prostate cancer cells (10). The present study aimed to examine the detailed function of the $\mathrm{NF}-\kappa \mathrm{B}$ pathway in osteosarcoma, and to determine whether punicalagin can inhibit the $\mathrm{NF}-\kappa \mathrm{B}$ pathway to suppress inflammation and osteosarcoma tumorigenicity. Combined treatment targeting the $\mathrm{NF}-\kappa \mathrm{B}$ pathway may represent a novel and promising strategy to significantly enhance the therapeutic activity of routine anticancer drugs against osteosarcoma.

\section{Materials and methods}

Reagents and cell lines. A $50 \mathrm{mM}$ stock solution consisting of $5 \mathrm{mg}$ punicalagin (Sigma-Aldrich; Merck KGaA) in $1 \mathrm{ml}$ DMSO were prepared. The stock was diluted to the desired concentrations with culture medium to give a water-soluble fraction, in which DMSO concentration did not exceed $0.2 \%$ in the highest punicalagin concentrations applied. The three human osteosarcoma cell lines (U2OS, MG63 and SaOS2) and one normal osteoblast cell line (hFOB1.19) were purchased from American Type Culture Collection and cultured according to the instructions. All of the cell lines were grown in Dulbecco's modified Eagle medium supplemented with 10\% FBS (both from Invitrogen; Thermo Fisher Scientific, Inc.) in a humidified atmosphere with $5 \% \mathrm{CO}_{2}$ at $37^{\circ} \mathrm{C}$. Following treatment, culture medium was prepared serum-free and collected in 24-h cultures. Phorbol myristate acetate (PMA; Sigma-Aldrich; Merck $\mathrm{KGaA}$ ), an activator of the NF- $\mathrm{NB}$ pathway, was added to cells together with punicalagin and incubated for $45 \mathrm{~min}$ in in a humidified atmosphere with $5 \% \mathrm{CO}_{2}$ at $37^{\circ} \mathrm{C}$.

Cell proliferation assay. Cell proliferation was examined using Cell Counting Kit-8 (CCK-8; Dojindo Molecular Technologies, Inc.). Cells were seeded at a density of $1 \times 10^{3}$ cells per well in 96-well plates, then treated with different concentrations of punicalagin or DMSO as control. Following 1-3 days of culture, $10 \mu \mathrm{l}$ CCK-8 reagent was added to each well and cells were cultured for $1 \mathrm{~h}$. A microplate reader (Bio-Rad Laboratories, Inc.) was used to measure the absorbance at $450 \mathrm{~nm}$.

Cell invasion assay. Transwell migration assays were performed with $8.0-\mu \mathrm{m}$ pore polycarbonate filter inserts (Corning, Inc.) coated with Matrigel ${ }^{\mathrm{TM}}$ (BD Biosciences) at room temperature for $1 \mathrm{~h}$ before use. Briefly, osteosarcoma cells in $100 \mu \mathrm{M}$ punicalagin contained or vehicle medium supplemented with $1 \%$ FBS were placed in the top chamber at a density of $1 \times 10^{4}$ cells/well. In the bottom chamber, complete medium with $10 \%$ FBS was used as a positive control. After $48 \mathrm{~h}$ of incubation, the migrated cells were fixed with $4 \%$ paraformaldehyde for $20 \mathrm{~min}$ and stained with $1 \%$ crystal violet for $10 \mathrm{~min}$ at room temperature. Images were captured with a light microscope at $x 400$ magnification and the migrated cells were counted manually and averaged from 5 high-power fields.

Cell apoptosis analysis. To investigate early and late apoptotic cells, annexin V-FITC and propidium iodide (PI) double staining was performed. The APOAF annexin $\mathrm{V}$ apoptosis kit (Sigma-Aldrich; Merck KGaA) was used for annexin $\mathrm{V}$ staining, according to the manufacturer's protocol. All samples were quantified using a Canto II flow cytometer (BD Biosciences) and analyzed with FlowJo version 7.6 software (TreeStar). Early apoptotic cells were defined as FITC ${ }^{\text {high }} \mathrm{PI}^{\text {low }}$ cells and late apoptotic cells were defined as FITC ${ }^{\text {high }}$ PI $^{\text {high }}$. Additionally, FITC ${ }^{\text {low }} \mathrm{PI}^{\text {low }}$ represented healthy cells and FITC ${ }^{\text {low }} \mathrm{PI}^{\text {high }}$ accounted for cells debris that was eliminated.

Western blotting assay. The cells or tissues were homogenized in RIPA buffer (Beyotime Institute of Biotechnology). A BCA protein assay kit (Thermo Fisher Scientific, Inc.) was used to determine the protein concentration in lysates and conditioned medium. Equal amounts of protein $(15 \mu \mathrm{g})$ were loaded per lane and separated via SDS-PAGE (10\% gel), then transferred to a PVDF membrane (Bio-Rad Laboratories, Inc.). The PVDF membrane was blocked with $5 \%$ skimmed milk in TBS + $0.1 \%$ Tween $^{\circledR}-20$ buffer on a shaker for $1 \mathrm{~h}$ at room temperature. The membrane was then incubated in $4^{\circ} \mathrm{C}$ with the following primary antibodies overnight: Anti-phosphorylated (phosphor)-inhibitor of $\kappa \mathrm{B} \alpha(\mathrm{I} \kappa \mathrm{B} \alpha$; Ser32; cat. no. 2859), anti-IкB $\alpha$ (cat. no. 9242), anti-phospho-mammalian target of rapamycin (mTOR; Ser2448, cat. no. 2971), anti-mTOR (cat. no. 2983), anti-p65 (cat. no. 8242), anti-histone 2A family member X (H2AX, cat. no. 7631) (all 1:1,000; all from Cell Signaling Technology Biological Reagents Co., Ltd.), anti- $\beta$-actin (cat.no. sc-130656; 1:2,000), anti-interleukin(IL)-6 (cat. no. sc-130326; 1:500), and anti-IL-8 (cat. no. sc-8427; 1:500; all purchased from Santa Cruz Biotechnology, Inc.). After washing, the membrane was incubated with horseradish peroxide-conjugated secondary antibody (cat. no 7074; 1:1,000 Cell Signaling Technology Biological Reagents Co., Ltd.) for $1 \mathrm{~h}$ on the shaker at room temperature. The membrane was then incubated with chemiluminescence reagent (GE Healthcare Life) for $5 \mathrm{~min}$ at room temperature. The relative quantity of the protein was measured using ImageJ software v1.51 (National Institutes of Health).

Tumor xenografts. The in vivo experiment protocol was approved by the Institutional Animal Care and Use Committee at The Second Affiliated Hospital of Air Force Medical University and followed the Chinese national standards: Laboratory animal welfare ethics review guidelines for the humane and customary care and use of experimental animals. A total of 20 female 6-8-week-old, $18 \mathrm{~g}$, Balb/c nude mice ( $n=5$ per group) were purchased from Model Animal Research Center of Nanjing University. Mice were housed at $20-24^{\circ} \mathrm{C}$ with an average humidity of $40 \%$ and a 12-h light/dark cycle and received food and water ad libitum. Mice were then injected with osteosarcoma cells near the back of the neck at a density of $2 \times 10^{7}$ cells in $200 \mu \mathrm{l}$ PBS. Mice were anesthetized by inhalation using 2.0-2.5\% sevoflurane during injection and measurement. After 1 week of tumor cell inoculation, $5 \mathrm{mg} / \mathrm{kg}$ punicalagin in saline or an equal volume $(300 \mu \mathrm{l})$ saline as vehicle (control) was injected intraperitoneally once a week for a total of 7 weeks, and the mouse health and behavior were monitored daily for 8 weeks. No death was observed prior to sacrifice. The tumor size was measured with a sliding caliper twice a week, and 
A

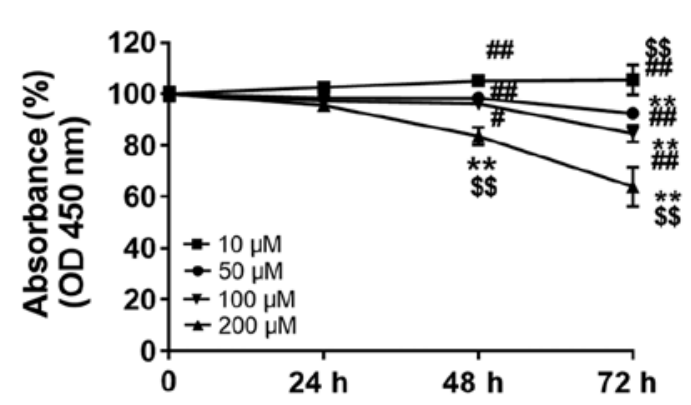

C

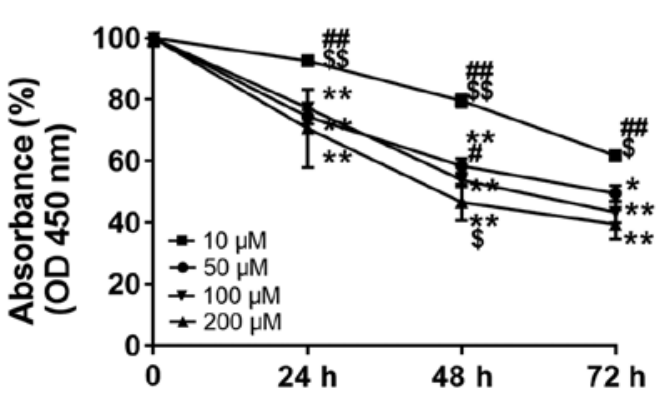

B

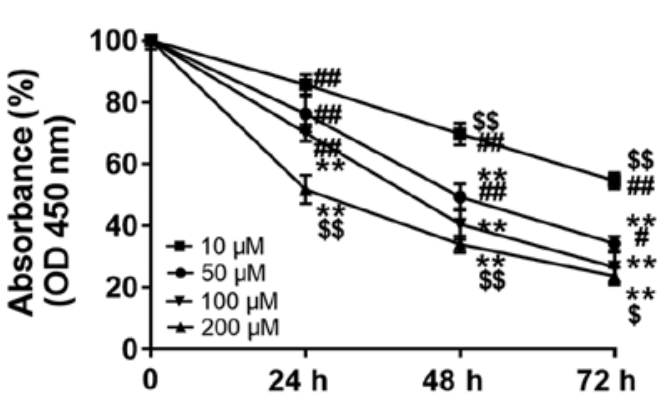

D

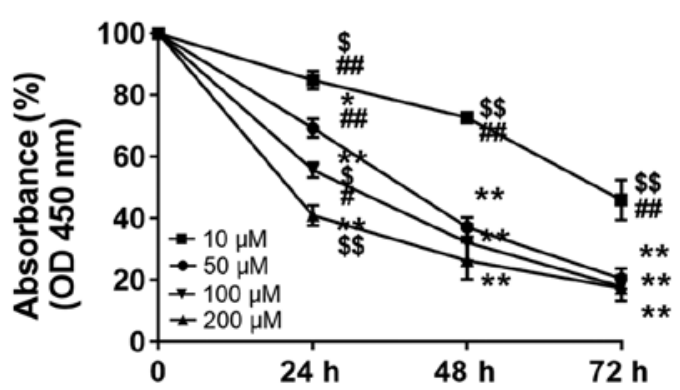

Figure 1. Effect of punicalagin on the viability of human osteoblast and osteosarcoma cell lines. Cells were treated with different concentrations of punicalagin for 24-72 h. Absorbance measured using a CCK-8 assay relative to the untreated subgroup in (A) hFOB1.19, (B) U2OS, (C) MG63, and (D) SaOS2. Data are presented as the mean $\pm \mathrm{SEM}$ of three independent experiments. ${ }^{*} \mathrm{P}<0.05$ and ${ }^{* *} \mathrm{P}<0.01$ vs. $10 \mu \mathrm{M}$ punicalagin; ${ }^{\$} \mathrm{P}<0.05$ and ${ }^{\$ \$} \mathrm{P}<0.01$, vs. $50 \mu \mathrm{M}$ punicalagin; ${ }^{\#} \mathrm{P}<0.05$ and ${ }^{\# \#} \mathrm{P}<0.01$, vs. $200 \mu \mathrm{M}$ punicalagin. $\mathrm{OD}$, optical density.

the tumor volume was calculated using the formula: Size, $\left.\mathrm{mm}^{3}=[\text { tumor length } \mathrm{x} \text { (tumor width })^{2}\right] / 2$. When volume was $>500 \mathrm{~mm}^{3}$, the experiment was stopped and the mice were sacrificed using $\mathrm{CO}_{2}$ asphyxiation with a flow rate $\leq 50 \%$ of the chamber volume per min, followed by cervical dislocation. Tumors were then harvested, weighed and snap-frozen in liquid nitrogen and stored at $-80^{\circ} \mathrm{C}$ for subsequent use immunohistochemistry assays.

Immunohistochemistry assay. Solid tumors were fixed with $10 \%$ formaldehyde for $48 \mathrm{~h}$ at room temperature and embedded in paraffin. Tissue slides were blocked with $1 \%$ BSA (Beyotime Institute of Biotechnology) in PBS for blocking for $1 \mathrm{~h}$ at room temperature. To identify infiltrating blood vessels, immunohistochemistry was carried out on 5- $\mu \mathrm{m}$ deparaffinized sections using an anti-CD31 antibody (cat. no. 77699; 1:150; Cell Signaling Technology Biological Reagents Co., Ltd.) at $4^{\circ} \mathrm{C}$ overnight, and then peroxide-conjugated secondary antibodies (cat. no. ab6721; 1:500; Abcam) for $1 \mathrm{~h}$ at room temperature with ABC Staining kits (Thermo Fisher Scientific, Inc.) were applied for generating chromogenic signals. Images were captured with a light microscope at magnification, x100.

Statistical analysis. Data are presented as the mean \pm standard error of the mean (SEM), unless otherwise stated. Analysis of two independent groups was performed using unpaired Student's t-test. One-way ANOVA followed by Bonferroni correction was used for multiple comparisons between groups. Statistical analysis was carried out using the GraphPad software v5.0 (GraphPad, Inc.). Each experiment was performed in triplicate. $\mathrm{P}<0.05$ was considered to indicate a statistically significant difference.

\section{Results}

Punicalagin treatment inhibits the proliferation of human osteosarcoma cell lines. In order to investigate whether punicalagin treatment could affect the viability malignant cells, hFOB1.19 osteoblast cells and U2OS, MG63 and SaOS2 osteosarcoma cells were treated with increasing concentrations of punicalagin $(10,50,100$ and $200 \mu \mathrm{M})$ for 24-72 h. Cell viability was then evaluated using CCK-8 assays (Fig. 1). Cell proliferation was suspended after $24 \mathrm{~h}$ but the viability of osteoblast cells did not significantly decrease at punicalagin concentrations $<100 \mu \mathrm{M}$ or incubation time $<48 \mathrm{~h}$. Overall, the viability of human osteosarcoma cell lines was decreased in a concentration- and time-dependent manner. In 2 of the 3 (MG2 and SaOS2) osteosarcoma cell lines, the decrease in cell viability was not further exacerbated following prolonged treatment $>48 \mathrm{~h}$ and concentrations $>100 \mu \mathrm{M}$. Thus, a concentration of $100 \mu \mathrm{M}$ was selected for subsequent experimentation.

Punicalagin increases apoptosis of human osteosarcoma cell lines. In order to determine whether the decrease in cell viability following treatment with a moderate concentration of punicalagin was due to increased apoptosis, annexin V-FITC and PI double staining was used to assess the frequency of early (FITC ${ }^{\text {high }} \mathrm{PI}^{\text {low }}$ ) and late (FITC $\left.{ }^{\text {high }} \mathrm{PI}^{\text {high }}\right)$ apoptotic cells in U2OS, MG63 and SaOS2 cell lines. Treatment with $100 \mu \mathrm{M}$ punicalagin for $48 \mathrm{~h}$ increased the cumulative percentage of early and late apoptotic tumor cells (Fig. 2). 
A
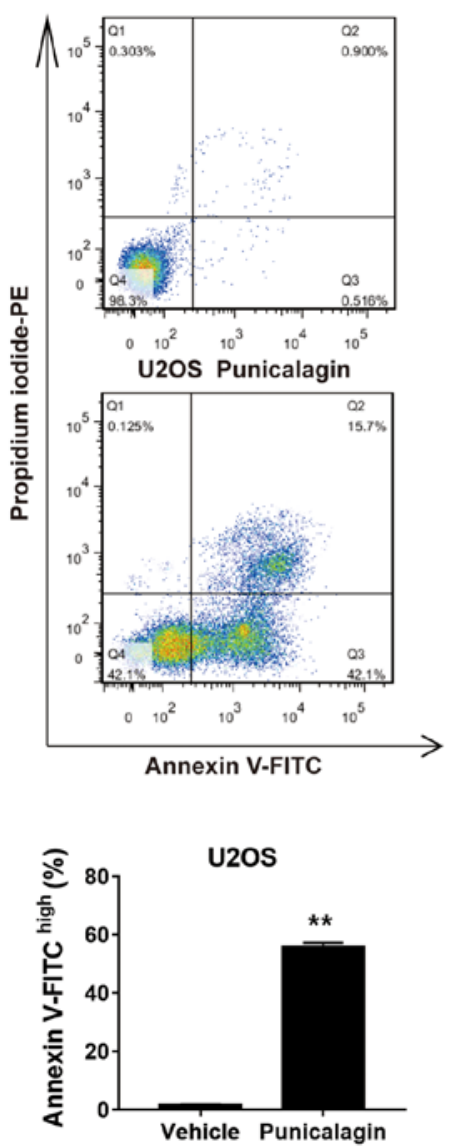

B
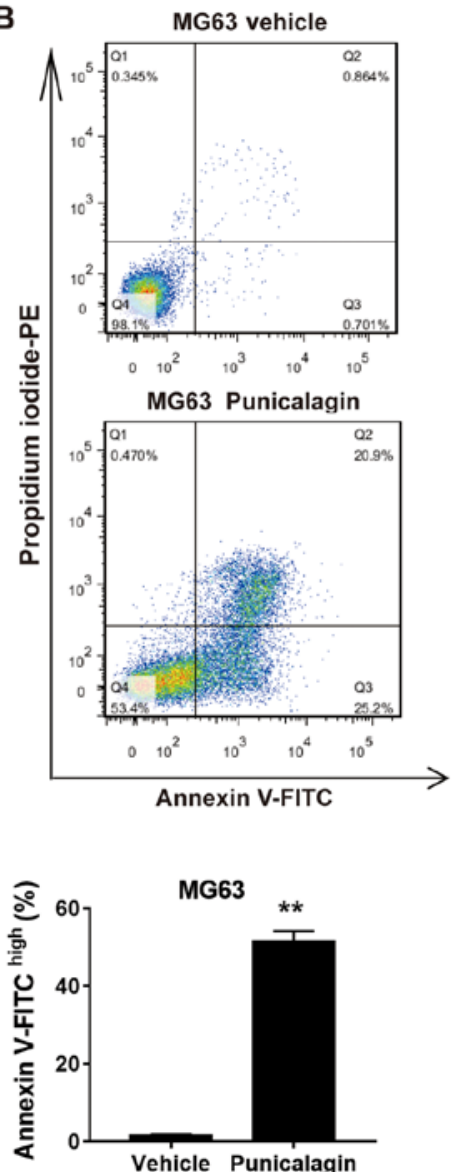

C
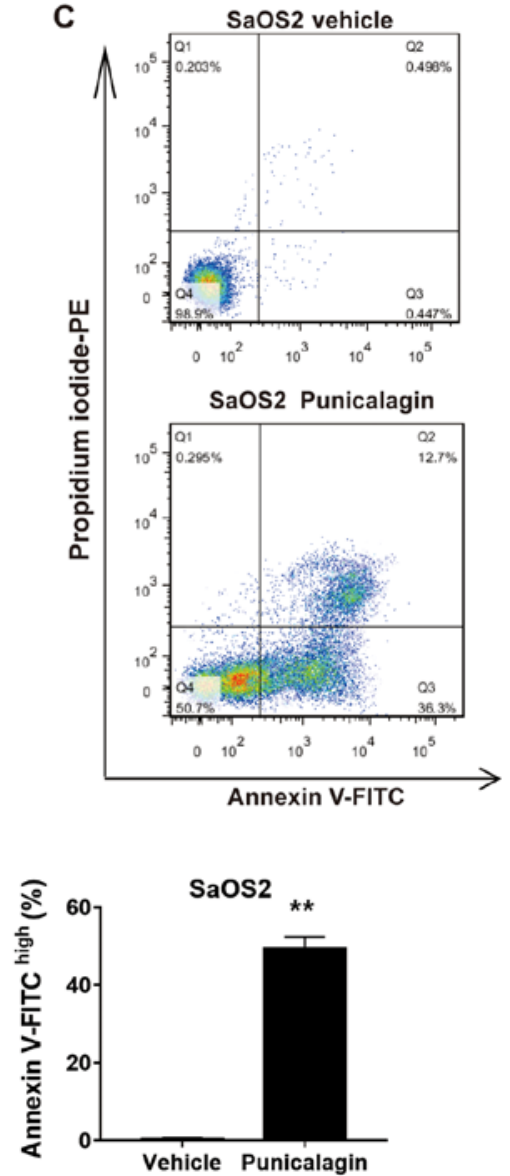

Figure 2. Effect of punicalagin on the apoptosis of human osteosarcoma cell lines. Cells were treated with $100 \mu \mathrm{M}$ punicalagin for $48 \mathrm{~h}$, then analyzed using annexin $\mathrm{V}$ and propidium iodide double staining. Punicalagin treatment induced consistent changes seen as increased frequencies of both early (FITChighPIlow) and late (FITChighPIhigh) apoptotic cells in (A) U2OS, (B) MG63 and (C) SaOS2 cells. The representative diagrams of flow cytometry analyses were displayed. Data are presented as the mean \pm SEM of three independent experiments. ${ }^{* *} \mathrm{P}<0.01$, vs. control group. PE, phycoerythrin; FITC, Fluorescein isothiocyanate; Ctrl, control.

A
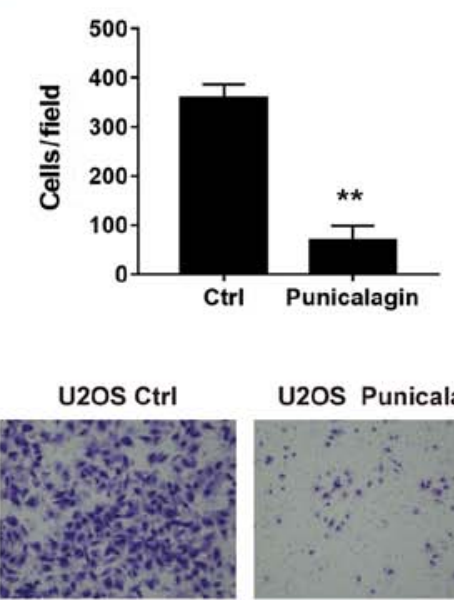

U2OS

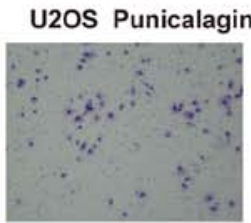

B

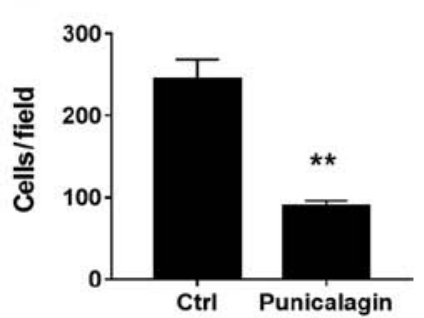

MG63 Ctrl

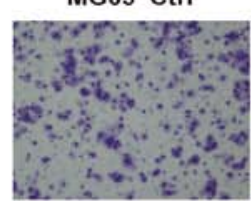

MG63 Punicalagin

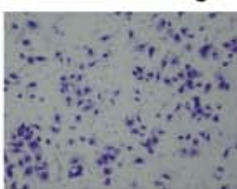

C

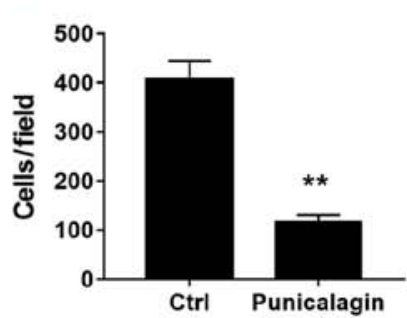

SaOS2 Ctrl SaOS2 Punicalagin
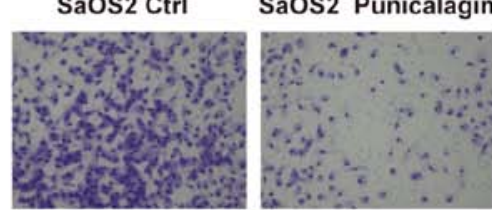

Figure 3. Effect of punicalagin on the invasion of human osteosarcoma cell lines (A) U2OS, (B) MG63 (B), and (C) SaOS2. Cells were treated with punicalagin for $24 \mathrm{~h}$, then analyzed using a Transwell invasion assay. Data are expressed as the mean \pm SEM of three independent experiments. ${ }^{* *} \mathrm{P}<0.01$, vs. control group. Ctrl, control.

Punicalagin suppresses the invasion of human osteosarcoma cells. To determine whether punicalagin could suppress the invasiveness of osteosarcoma cells, a Transwell Matrige ${ }^{\mathrm{TM}}$ invasion assay was performed using U2OS, MG63 and SaOS2 cells in the presence or absence of punicalagin. Following treatment with punicalagin for $24 \mathrm{~h}$, fewer migrated tumor cells were detected, suggesting that cell migration was inhibited in the presence of punicalagin (Fig. 3). 
A

A Punicalagin
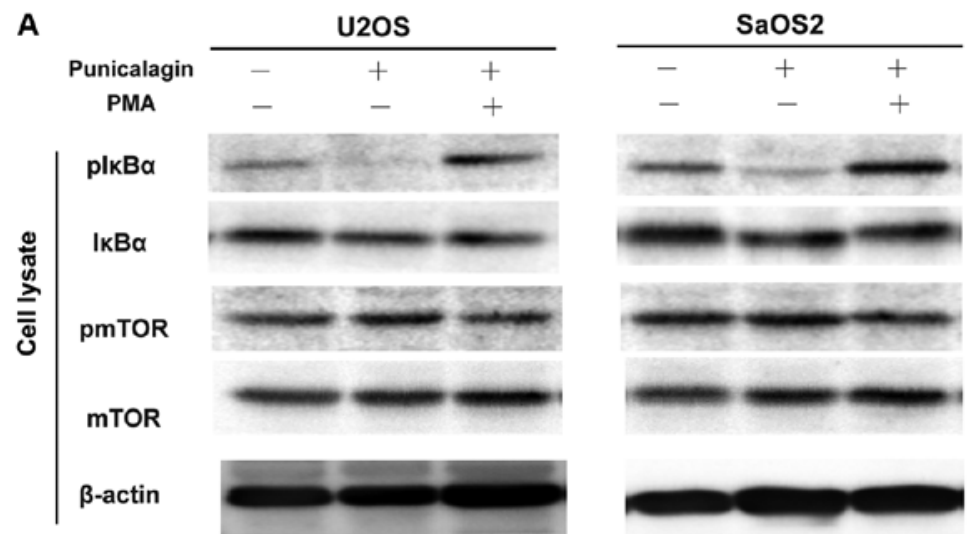

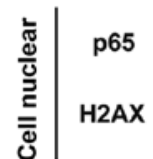
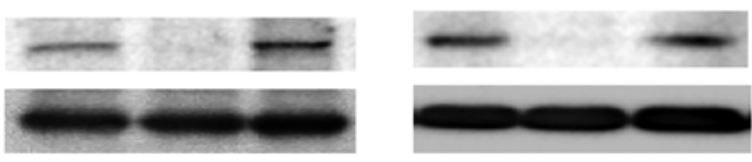

B

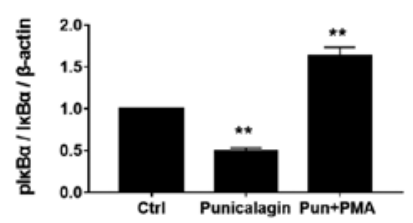

U2OS

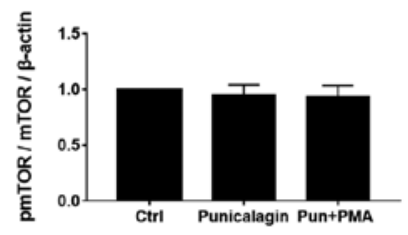

SaOS2
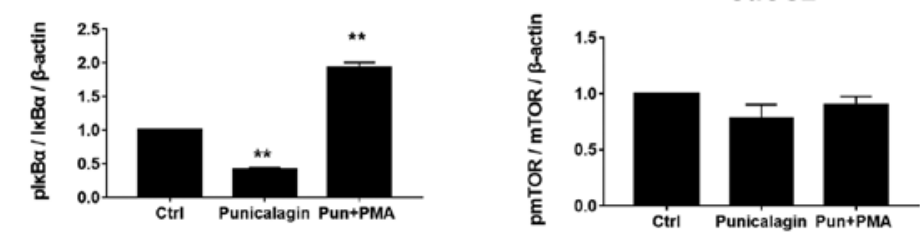
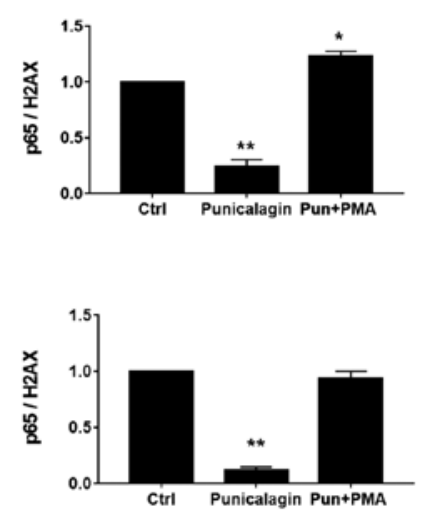

。

(1)

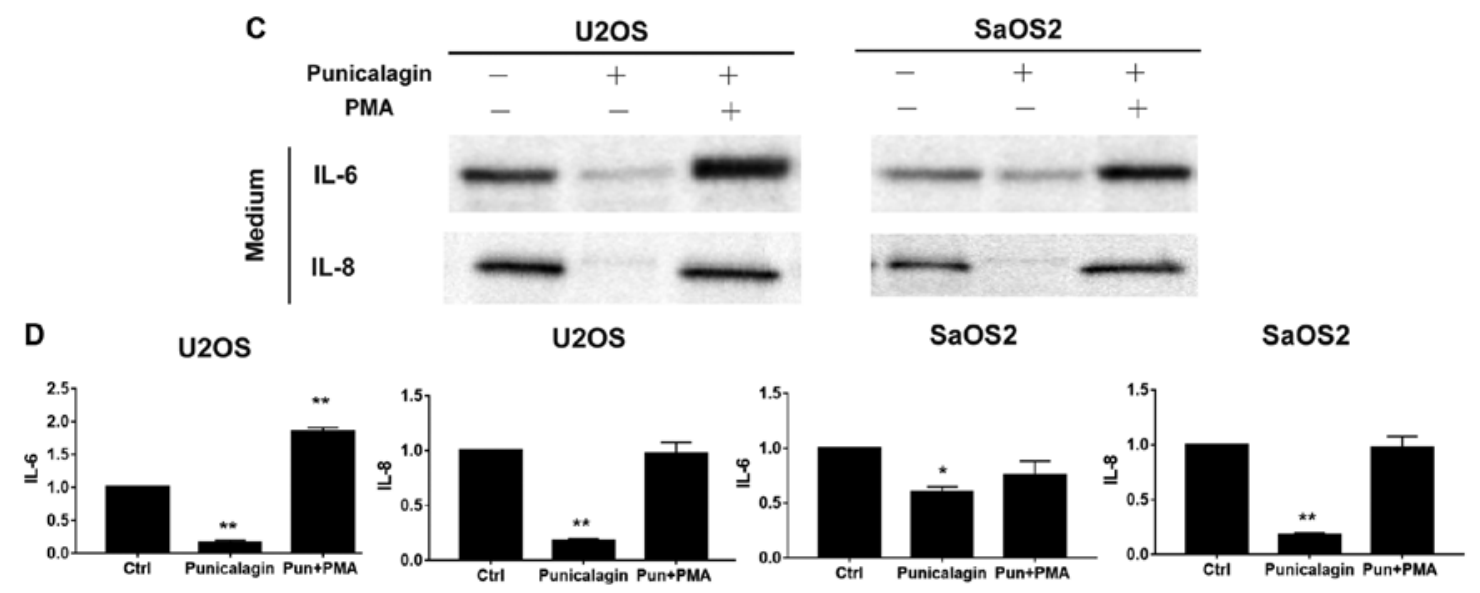

Figure 4. Punicalagin regulates the NF-кB pathway in human osteosarcoma cells. (A) Total cell extracts or nuclear fractions were collected and subjected to western blot analysis using anti-mTOR, pmTOR (Ser2448), IкB $\alpha, \mathrm{pI \kappa \textrm {B }} \alpha$ (Ser32), $\beta$-actin, nuclear p65 and H2AX. (B) Quantification of the results shown in (A). (C) U2OS and SaOS2 cells were also treated with punicalagin solution. The conditioned medium with or without NF- $\kappa$ B signaling activator, PMA and punicalagin treatment were used to determine the levels of IL-6 and IL-8. (D) Quantification of the results shown in (C). ${ }^{*} \mathrm{P}<0.05$ and ${ }^{* *} \mathrm{P}<0.01$ vs. control group.

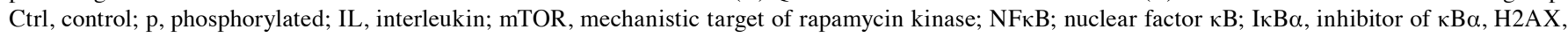
histone 2A family member X; PMA, phorbol myristate acetate; Pun, punicalagin.

Punicalagin downregulates the $N F-\kappa B$, but not mTOR signaling pathway in osteosarcoma cell lines. AKT signaling regulates cell survival. As one of the main downstream mediators, mTOR signaling is essential for cell proliferation, and suppressed mTOR signaling is associated with apoptosis induction by various stimuli, delayed cell cycle progression and cell proliferation (11). The levels of phospho-mTOR/mTOR expression in osteosarcoma cells lysates were not altered by punicalagin treatment, but compared with those of the control group the levels of 

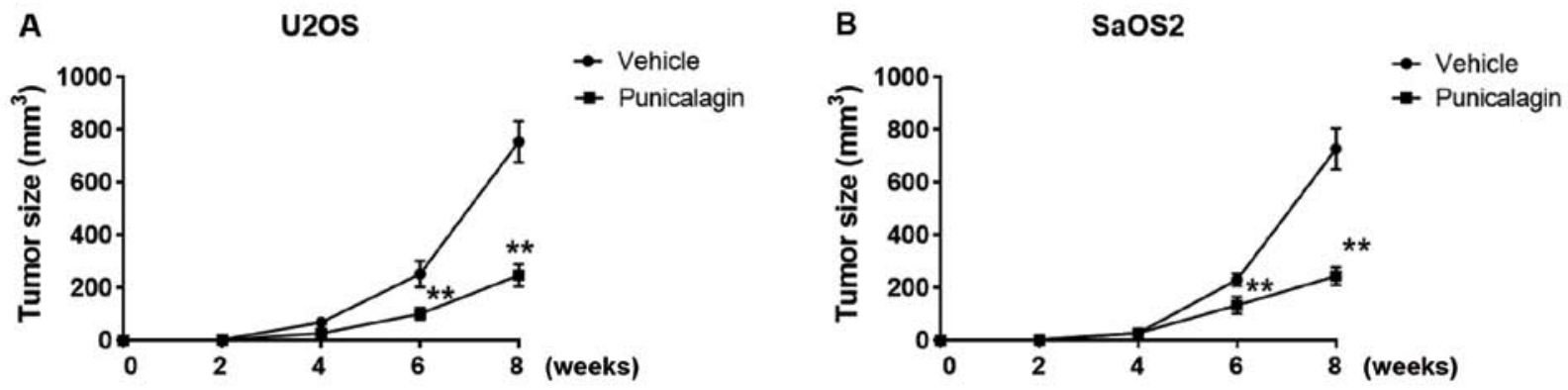

C

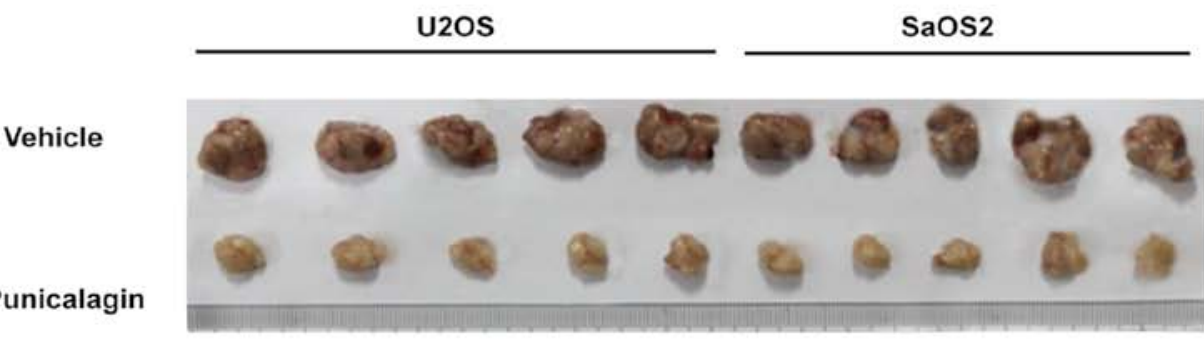

D

U2OS

E

SaOS2
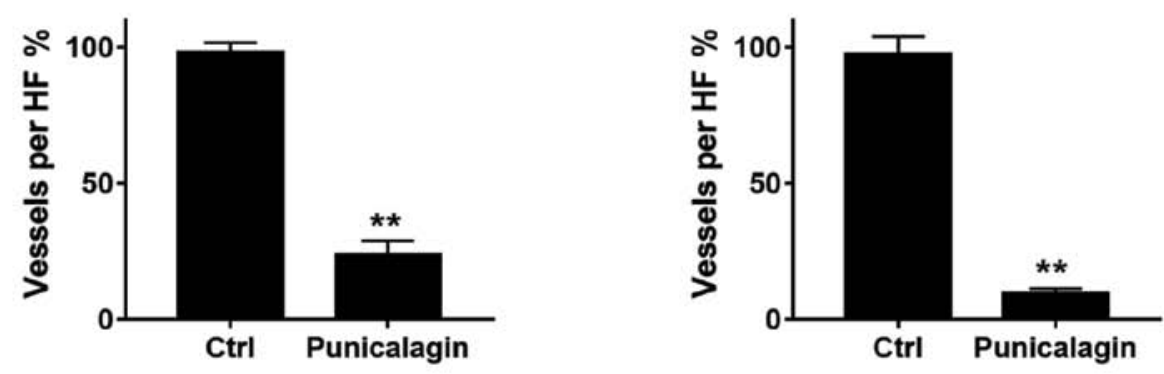

F

U2OS
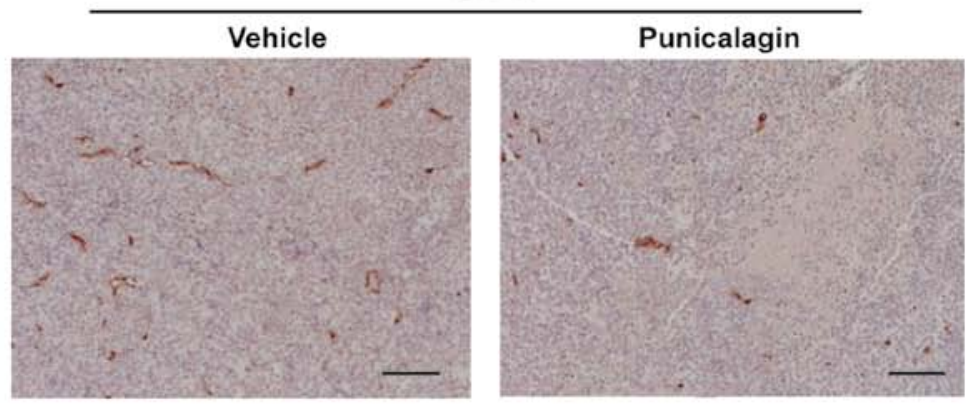

SaOS2
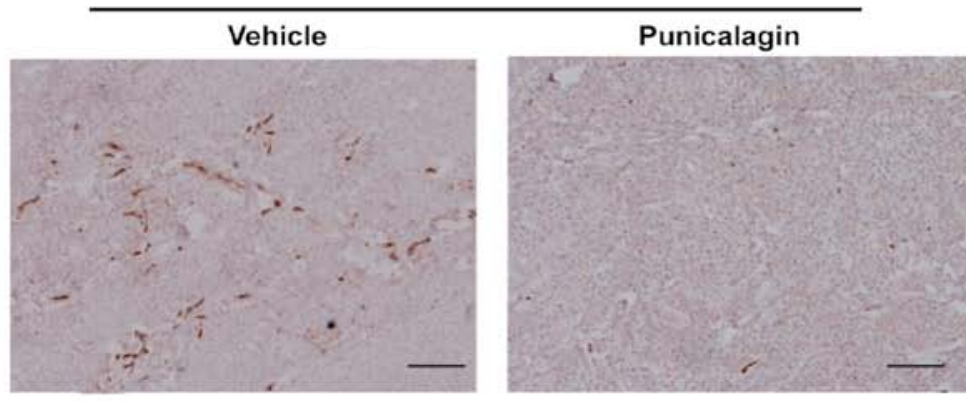

Figure 5. Punicalagin attenuates the malignant growth of osteosarcoma cells in mice. (A and B) Xenografted osteosarcoma growth curves of (A) U2OS and (B) SaOS2 were performed according to the xenograft tumor volume. (C) The representative osteosarcoma tissues after resection in mice xenograft models were captured. (D and E) Quantification of the angiogenesis results in xenografted osteosarcoma of (D) U2OS and (E) SaOS2 cells. (F) Punicalagin inhibits tumor angiogenesis represented by decreased blood vessel staining. ${ }^{* *} \mathrm{P}<0.01$ vs. vehicle group. Blood vessels are indicated by CD31 expression stained brown. Scale bar, $10 \mu \mathrm{m}$. Ctrl, control. 
$\mathrm{pI} \kappa \mathrm{B} \alpha / \mathrm{I} \kappa \mathrm{B} \alpha, \mathrm{p} 65$, IL-6, IL- 8 were significantly reduced by punicalagin treatment (Fig. 4).

$\mathrm{NF}-\kappa \mathrm{B}$ is a key regulator of inflammatory immune responses, including cytokine production (12). During tumorigenesis, these cytokine-associated chemotactic effects are required for the initiation of tumor-associated inflammation and neovascularization (13). Osteosarcoma cells were treated with saline (vehicle) or $100 \mu \mathrm{M}$ punicalagin alone for $48 \mathrm{~h}$, following which, changes in the activated levels of $\mathrm{NF}-\kappa \mathrm{B}$ represented by $\mathrm{pI} \kappa \mathrm{B} \alpha / \mathrm{I} \kappa \mathrm{B} \alpha$ and $\mathrm{p} 65$, and its downstream inflammatory factors, including IL-6, IL-8 were examined (Fig. 4). Punicalagin affected the stable expression of $\mathrm{NF}-\kappa \mathrm{B}$ in U2OS and SaOS2 cells. The expression levels of phosphor-I $\kappa \mathrm{B} \alpha$, nuclear $\mathrm{p} 65$ and IL-6, IL-8 significantly decreased in U2OS and SaOS2 cells compared with untreated cells. To further evaluate the effect of punicalagin on the NF- $\kappa$ B pathway, $200 \mathrm{nM}$ PMA, an activator of the NF- $\kappa$ B pathway, was added and cells were incubated for 45 min. Following the addition of PMA, the downregulation of IL-6 and IL-8 levels observed in punicalagin pre-treated osteosarcoma cells was reversed.

Punicalagin attenuates proliferation and angiogenesis of osteosarcoma cells in a murine tumor xenograft model. Proliferation and migration of osteosarcoma cells were significantly attenuated in a xenograft model in mice. Tumors typically had a length of 11-18 mm and a width of $8-12 \mathrm{~mm}$ in the vehicle group. By contrast, punicalagin treatment significantly decreased tumor growth, to a length of 7-11 mm and width of 6-8 mm (Fig. 5A-C). Thus, punicalagin injection resulted in slower malignant growth of human osteosarcoma cells in the mouse model in vivo. Staining of blood vessels with CD31 antibodies was then used to evaluate angiogenesis in malignant tissues of xenograft mice. CD31 staining represented the wall of the blood vessel of osteosarcoma core sections that were used in a rat subcutaneous model, as described in a previous study (14). The mean density of blood vessels in the punicalagin-treated was significantly reduced, compared with that in the vehicle group, which suggested that that punicalagin could inhibit tumor angiogenesis (Fig. 5D-F).

\section{Discussion}

The present study suggested that punicalagin treatment in osteosarcoma cells significantly decreased tumor cell viability and induced cell apoptosis. Punicalagin can inhibit proliferation and survival of osteosarcoma cells in a concentration- and time-dependent manner. These results were also replicated in a xenograft model, in which impaired angiogenesis was also observed following injection of punicalagin. The molecular mechanisms were further investigated using biological methods, which demonstrated that the therapeutic effects of punicalagin were associated with downregulation of $N F-\kappa B$ but not mTOR signaling.

Osteosarcoma is the most commonly diagnosed primary solid bone malignancy. Metastasis is the main cause of death in patients with osteosarcoma, and treatment options remain unsatisfactory. The incidence of osteosarcoma in the general population is 2-3 per million per year. However, annual incidence is around 1.2-7.6 per million per year in people younger than 24 years of age worldwide (15). Although major efforts have been made to establish the potential pathognomonic driver mutations in young patients, only sporadic mutations have detected in great majority of cases. Similar to the majority of common types of human cancer, osteosarcoma exhibits a high degree of mutational diversity. This diversity is driven by complex rearrangement processes, chromothripsis and chromothripsic amplification that predominate in osteosarcoma, although the underlying causes remain unknown (16). Currently, early detection, quick confirmation or targeted therapeutic strategies by molecular biology are not available in clinical practice. Therefore, the present study aimed to investigate the efficacy of a potential anti-cancer compound, punicalagin, in osteosarcoma cells.

Although they represent the main available treatment in osteosarcoma, chemotherapeutics are also toxic to normal tissue, and can lead to myelosuppression, opportunistic infection, heart damage and other adverse reactions, thereby decreasing the patient survival rate and quality of life (17). Therefore, new agents with fewer side effects and improved therapeutic advantages are required. Block et al (18) suggested that a phytochemical-rich diet, which includes compounds such as polyphenols, salicylates, phytosterols, saponins, glucosinolates, protease inhibitors, monoterpenes, terpenes, lectins, was associated with decreased risk of cancer. Punicalagin is one of the most abundant polyphenols in pomegranate. In addition, increasing evidence suggests that punicalagin inhibits tumor invasion and metastasis of various types of cancer, such as cervical (19), ovarian (20), colon (21) and lung cancer (22) as well as antioxidants in chronic inflammation (23). To the best of our knowledge, the present study was the first to identify that punicalagin could significantly inhibit osteosarcoma cell proliferation and invasion, induce apoptosis, and decrease angiogenesis. Thus, the results of the present study may provide insight into future therapeutic strategies against osteosarcoma.

However, as previously shown in both rats and humans, the poor bioavailability of punicalagin represents a considerable limitation to pharmaceutical research on its potential therapeutic effects in vivo (24). The low bioavailability of ellagic acid generated from punicalagin is due to its hydrophilic structure and large molecular weight, which limits its absorption by simple diffusion, including oral administration (25). In addition, extremely low lipid solubility further restricts its permeability through the lipophilic layer of the gastrointestinal tract (26). Furthermore, punicalagin can be metabolized into the bioavailable but relatively poor antioxidant, hydroxy-6H-dibenzopyran-6-one derivatives by the colonic microflora in healthy humans (27). Based on animal studies, the serum concentration after absorption of punicalagin in rodents was $\sim 30 \mu \mathrm{M}$ (28), which is lower than the concentration used in vitro in the present study. Thus, the multifaceted therapeutic benefits of punicalagin observed in the present study may be difficult to fully replicate in patients. Furthermore, a relatively high concentration of punicalagin may result in non-specific effects due to the biological differences between osteoblasts and osteosarcoma cells. However, the development of novel punicalagin derivatives, compound preparation and administration methods may overcome these limitations in the future.

The precise mechanisms through which punicalagin inhibits osteosarcoma invasion and angiogenesis, as well 
as its regulation, are not well understood. Several previous studies demonstrated that multiple signaling pathways, including the MAPK (29), $\beta$-catenin (19), TGF- $\beta 1$ (30), AKT, and JNK (31) pathways, were modulated by punicalagin administration. Furthermore, Adams et al (32) suggested that punicalagin decreased phosphorylation of the $\mathrm{p} 65$ subunit and binding of $\mathrm{NF}-\mathrm{\kappa B}$ about 3.6-fold in colon cancer. In nerve cells, chronic neuroinflammation and oxidative stress were dramatically diminished by punicalagin via NF- $\kappa \mathrm{B}$ inhibition (33). Furthermore, vascular endothelial growth factor, an $\mathrm{NF}-\kappa \mathrm{B}$ transcriptional target gene, was downregulated by punicalagin, thereby decreasing angiogenesis in the tumor environment $(34,35)$. The in vitro and in vivo results of the present study were consistent with previous reports, and demonstrated the therapeutic potential of punicalagin against mesodermal illness likes osteosarcoma, through modulation of NF- $\kappa \mathrm{B}$ activity.

In general, $\mathrm{NF}-\kappa \mathrm{B}$ signaling controls many cellular processes, including immune responses, immune cell proliferation and viability, lymphogenesis and $\mathrm{B}$ cell maturation (36). The NF- $\mathrm{B}$ pathway is also involved in the regulation of skeletal muscle cell differentiation (37). Recently, activation of NF- $\kappa \mathrm{B}$ was demonstrated to increase glucose uptake and glycolytic flux in sarcoma cells, which suggested that $\mathrm{NF}-\kappa \mathrm{B}$ played a crucial role in the development of osteosarcoma malignancies (38). Consistently, Gong et al (39) found at least $75 \%$ osteosarcoma tissues from patients showed positive stain of activated NF- $\kappa$ B pathways and patients whose osteosarcoma with active NF- $\kappa \mathrm{B}$ had short median overall survival time as compared with patients whose osteosarcoma had inactive NF- $\mathrm{NB}$. Expression of metastasis-associated proteins, angiogenesis, cell invasion and metastasis have also been linked to NF- $\mathrm{kB}$ activation in osteosarcoma (8). Liao et al (40) used short hairpin RNA to knockdown NF-kB expression, which abolished cell invasion and metastasis in osteosarcoma. In another previous study, the NF-KB inhibitor QNZ suppressed NF-kB activation, which resulted in downregulation of proteins associated with metastasis, cell migration and cell invasion in osteosarcoma cells (41). The present study further demonstrated that NF- $\kappa \mathrm{B}$ is an important transcription factor during pathogenesis of osteosarcoma, and that punicalagin was involved in modulating the expression of molecules downstream of NF- $\mathrm{B}$, such as IL-6, and IL-8.

A previous study demonstrated that IL- 6 and IL-8 genes were directly regulated by the $\mathrm{NF}-\kappa \mathrm{B}$ pathway (42) and that IL- 6 and IL-8 levels increased with NF- $\kappa \mathrm{B}$ overexpression during chronic inflammation in bone and joint tissues (43). IL-6 and IL-8 activation promotes an inflammatory microenvironment during malignant progression (44) and these cytokine-associated chemotactic effects are required for the initiation of tumor-associated inflammation and neovascularization (45). The present study indicated that punicalagin decreased IL-6 as well as IL-8 production by osteosarcoma cells, which was consistent with angiogenesis inhibition in xenograft models. Thus, these findings further elucidate the mechanisms underlying the preventive and therapeutic potential of punicalagin against osteosarcoma.

Although a previous study suggested that pomegranate extract, including a large amount of active punicalagin, had a strong anti-aging effect through the mTOR pathway (46), the present study failed to confirm this finding. Thus, the present results highlight the significance of punicalagin as a promising tumor suppressor in osteosarcoma by targeting NF- $\kappa \mathrm{B}$, but not mTOR pathway. Further characterization of this compound will provide a new insight into punicalagin-mediated suppression of osteosarcoma genesis and development.

In conclusion, punicalagin treatment inhibited osteosarcoma growth, including proliferation, invasion and angiogenesis through $\mathrm{NF}-\kappa \mathrm{B}$ suppression. Further in-depth and long-term studies are required in order to establish the therapeutic target of the NF- $\mathrm{BB}$ signaling pathway in punicalagin-induced cell survival and inhibition of invasive abilities, as well as excessive angiogenesis. Nonetheless, the results of our present study provide preliminary evidence to support punicalagin, a phytochemical used in herbal medicine, as a novel and effective candidate for the systemic treatment and/or chemoenhancement of osteosarcoma.

\section{Acknowledgements}

Not applicable.

\section{Funding}

No funding was received.

\section{Availability of data and materials}

All data generated or analyzed during this study are included in this published article.

\section{Authors' contributions}

HW designed and directed the study, and analyzed and interpreted the data. TH performed the experiments and wrote the manuscript. XZ performed the literature search, analyzed the data and designed the figures. All authors read and approved the final manuscript.

\section{Ethics approval and consent to participate}

The present study was approved by The Animal Ethics Committee of The Second Affiliated Hospital of Air Force Medical University (approval no. 201904-11).

\section{Patient consent for publication}

Not applicable.

\section{Competing interests}

The authors declare that they have no competing interests.

\section{References}

1. Damron TA, Ward WG and Stewart A: Osteosarcoma, chondrosarcoma, and Ewing's sarcoma: National cancer data base report. Clin Orthop Relat Res 459: 40-47, 2007.

2. Anderson ME: Update on survival in osteosarcoma. Orthop Clin North Am 47: 283-292, 2016. 
3. Geller DS and Gorlick R: Osteosarcoma: A review of diagnosis, management, and treatment strategies. Clin Adv Hematol Oncol 8: 705-718, 2010.

4. Ottaviani $\mathrm{G}$ and Jaffe $\mathrm{N}$ : The epidemiology of osteosarcoma (M)//Jaffe N, Bruland OS, Bielack S (Eds.). Pediatric and adolescent osteosarcoma. Boston, MA; Springer US pp. 3-13, 2010.

5. Isakoff MS, Bielack SS, Meltzer P and Gorlick R: Osteosarcoma: Current treatment and a collaborative pathway to success. J Clin Oncol 33: 3029-3035, 2015.

6. Bielack SS, Smeland S, Whelan JS, Marina N, Jovic G, Hook JM, Krailo MD, Gebhardt M, Pápai Z, Meyer J, et al: Methotrexate, doxorubicin, and cisplatin (MAP) plus maintenance pegylated interferon Alfa- $2 b$ versus MAP alone in patients with resectable high-grade osteosarcoma and good histologic response to preoperative MAP: First results of the EURAMOS-1 good response randomized controlled trial. J Clin Oncol 33: 2279-2287, 2015.

7. Chang J, Wang Z, Tang E, Fan Z, McCauley L, Franceschi R, Guan K, Krebsbach HP and Wang C: Inhibition of osteoblastic bone formation by nuclear factor-kappaB. Nat Med 15: 682-689, 2009.

8. Avnet S, Di Pompo G, Chano T, Errani C, Ibrahim-Hashim A, Gillie RJ, Donati DM and Baldini N: Cancer-associated mesenchymal stroma fosters the stemness of osteosarcoma cells in response to intratumoral acidosis via NF- $\kappa \mathrm{B}$ activation. Int J Cancer 140: 1331-1345, 2017.

9. Mongre RK, Sodhi SS, Ghosh M, Kim JH, Kim N, Sharma N and Jeong DK: A new paradigm to mitigate osteosarcoma by regulation of MicroRNAs and suppression of the NF- $\kappa \mathrm{B}$ signaling cascade. Dev Reprod 18: 197-212, 2014

10. Tang QL, Xie XB, Wang J, Chen Q, Han AJ, Zou CY, Yin JQ, Liu DW, Liang Y, Zhao ZQ, et al: Glycogen synthase kinase-3 $\beta$, $\mathrm{NF}-\kappa \mathrm{B}$ signaling, and tumorigenesis of human osteosarcoma. J Natl Cancer Inst 104: 749-763, 2012.

11. Testa JR and Tsichlis PN: AKT signaling in normal and malignant cells. Oncogene 24: 7391-7393, 2005.

12. Liu T, Zhang L, Joo D and Sun SC: NF- $\mathrm{BB}$ signaling in inflammation. Signal Transduct Target Ther 2: 17023, 2017.

13. Germano G, Allavena P and Mantovani A: Cytokines as a key component of cancer-related inflammation. Cytokine 43: 374-379, 2008.

14. Peng N, Gao S, Guo X, Wang G, Cheng C, Li M and Liu K: Silencing of VEGF inhibits human osteosarcoma angiogenesis and promotes cell apoptosis via VEGF/PI3K/AKT signaling pathway. Am J Transl Res 8: 1005, 2016.

15. Mirabello L, Troisi RJ and Savage SA: International osteosarcoma incidence patterns in children and adolescents, middle ages and elderly persons. Int J Cancer 125: 229-234, 2009.

16. Behjati S, Tarpey PS, Haase K, Ye H, Young MD, Alexandrov LB Farndon SJ, Collord G, Wedge DC, Martincorena I, et al: Recurrent mutation of IGF signalling genes and distinct patterns of genomic rearrangement in osteosarcoma. Nat Commun 8: 15936, 2017.

17. Zhang B, Zhang Y, Li R, Li J, Lu X and Zhang Y: The efficacy and safety comparison of first-line chemotherapeutic agents (high-dose methotrexate, doxorubicin, cisplatin, and ifosfamide) for osteosarcoma: A network meta-analysis. J Orthop Surg Res 15: 51, 2020.

18. Block G, Patterson B and Subar A: Fruit, vegetables, and cancer prevention: A review of the epidemiological evidence. Nutr Cancer 18: 1-29, 1992

19. Tang J, Li B, Hong S, Liu C, Min J, Hu M, Li Y, Liu Y and Hong L: Punicalagin suppresses the proliferation and invasion of cervical cancer cells through inhibition of the $\beta$-catenin pathway. Mol Med Rep 16: 1439-1444, 2017.

20. Tang JM, Min J, Li BS, Hong SS, Liu C, Hu M, Li Y, Yang J and Hong L: Therapeutic effects of punicalagin against ovarian carcinoma cells in association with $\beta$-Catenin signaling inhibition. Int J Gynecol Cancer 26: 1557-1563, 2016.

21. Omar U, Aloqbi A, Yousr M and Howell NK: Effect of punicalagin on human colon cancer caco-cells. Malaysian J Nutri 22 125-136, 2016

22. Li Y, Yang F, Zheng W, Hu M, Wang J, Ma S, Deng Y, Luo Y, Ye $\mathrm{T}$ and Yin W: Punica granatum (pomegranate) leaves extract induces apoptosis through mitochondrial intrinsic pathway and inhibits migration and invasion in non-small cell lung cancer in vitro. Biomed Pharmacother 80: 227-235, 2016.

23. Aloqbi A, Omar U, Yousr M, Grace M, Lila MA and Howell N: Antioxidant activity of pomegranate juice and punicalagin. Nat Sci 8: 235-246, 2016.

24. Mertens-Talcott SU, Jilma-Stohlawetz P, Rios J, Hingorani L and Derendorf H: Absorption, metabolism, and antioxidant effects of pomegranate (Punica granatum L.) polyphenols after ingestion of a standardized extract in healthy human volunteers. J Agric Food Chem 54: 8956-8961, 2006.
25. Vora A, Londhe V and Pandita N: Herbosomes enhance the in vivo antioxidant activity and bioavailability of punicalagins from standardized pomegranate extract. J Funct Foods 12: 540-548, 2015.

26. Seeram NP, Lee R and Heber D: Bioavailability of ellagic acid in human plasma after consumption of ellagitannins from pomegranate (Punica granatum L.) juice. Clin Chim Acta 348: 63-68, 2004.

27. Cerdá B, Espín JC, Parra S, Martínez P and Tomás-Barberán FA: The potent in vitro antioxidant ellagitannins from pomegranate juice are metabolised into bioavailable but poor antioxidant hydroxy-6H-dibenzopyran-6-one derivatives by the colonic microflora of healthy humans. Eur J Nutr 43: 205-220, 2004.

28. Cerdá B, Llorach R, Cerón JJ, Espín JC and Tomás-Barberán FA: Evaluation of the bioavailability and metabolism in the rat of punicalagin, an antioxidant polyphenol from pomegranate juice. Eur J Nutr 42: 18-28, 2003

29. Chu G, Zhang W, Chen M, Yang H and Yuan Z: Punicalagin inhibits RANKL-induced osteoclastogenesis by suppressing NF- $\kappa \mathrm{B}$ and MAPK signaling pathways. Int J Clin Exp Med 11: 6571-6582, 2018

30. Tang J, Liu C, Min J, Hu M, Li Y and Hong L: Potential therapeutic role of punicalagin against mechanical-trauma-induced stress urinary incontinence via upregulation of Nrf2 and TGF- $\beta 1$ signaling. Int Urogynecol J 28: 947-955, 2017.

31. Iwatake M, Okamoto K, Tanaka T and Tsukuba T: Punicalagin attenuates osteoclast differentiation by impairing NFATc1 expression and blocking Akt-and JNK-dependent pathways. Mol Cell Biochem 407: 161-172, 2015.

32. Adams LS, Seeram NP, Aggarwal BB, Takada Y, Sand D and Heber D: Pomegranate juice, total pomegranate ellagitannins, and punicalagin suppress inflammatory cell signaling in colon cancer cells. J Agric Food Chem 54: 980-985, 2006

33. Kim YE, Hwang CJ, Lee HP, Kim CS, Son DJ, Ham YW, Hellström M, Han SB, Kim HS, Park EK and Hong JT: Inhibitory effect of punicalagin on lipopolysaccharide-induced neuroinflammation, oxidative stress and memory impairment via inhibition of nuclear factor-kappaB. Neuropharmacology 117: 21-32, 2017.

34. Naugler WE and Karin M: NF-kappaB and cancer-identifying targets and mechanisms. Curr Opin Genet Dev 18: 19-26, 2008.

35. Toi M, Bando H, Ramachandran C, Melnick SJ, Imai A, Fife RS, Carr RE, Oikawa T and Lansky EP: Preliminary studies on the anti-angiogenic potential of pomegranate fractions in vitro and in vivo. Angiogenesis 6: 121-128, 2003.

36. Bakkar $\mathrm{N}$ and Guttridge DC: NF-kappaB signaling: A tale of two pathways in skeletal myogenesis. Physiol Rev 90: 495-511, 2010.

37. Bakkar N, Wang J, Ladner KJ, Wang H, Dahlman JM, Carathers M, Acharyya S, Rudnicki MA, Hollenbach AD and Guttridge DC: IKK/NF-kappaB regulates skeletal myogenesis via a signaling switch to inhibit differentiation and promote mitochondrial biogenesis. J Cell Biol 180: 787-802, 2008.

38. Londhe P, Yu PY, Ijiri Y, Ladner KJ, Fenger JM, London C, Houghton PJ and Guttridge DC: Classical NF- $\kappa$ B metabolically reprograms sarcoma cells through regulation of hexokinase 2 . Front Oncol 8: 104, 2018

39. Gong T, Su X, Xia Q, Wang J and Kan S: Expression of NF- $\kappa B$ and PTEN in osteosarcoma and its clinical significance. Oncol Lett 14: 6744-6748, 2017.

40. Liao CL, Lin JH, Lien JC, Hsu SC, Chueh FS, Yu CC, Wu PP, Huang YP, Lin JG and Chung JG: The crude extract of Corni Fructus inhibits the migration and invasion of U-2 OS human osteosarcoma cells through the inhibition of matrix metalloproteinase-2/-9 by MAPK signaling. Environ Toxicol 30: 53-63, 2015.

41. Pan PJ, Tsai JJ and Liu YC: Amentoflavone inhibits metastatic potential through suppression of ERK/NF- $\kappa \mathrm{B}$ activation in osteosarcoma U2OS cells. Anticancer Res 37: 4911-4918, 2017.

42. Hoesel B and Schmid JA: The complexity of NF-kappaB signaling in inflammation and cancer. Mol Cancer 12: 86, 2013.

43. Lv F, Song LJ, Wang XH, Qiu F and Li XF: The role of Act1, a NF- $\kappa \mathrm{B}$-activating protein, in IL-6 and IL-8 levels induced by IL-17 stimulation in SW982 cells. Pharm Biol 51: 1444-1450, 2013.

44. Karin M: NF- $\kappa B$ as a critical link between inflammation and cancer. Cold Spring Harb Perspect Biol 1: a000141, 2009.

45. Sparmann A and Bar-Sagi D: Ras-induced interleukin-8 expression plays a critical role in tumor growth and angiogenesis. Cancer Cell 6: 447-458, 2004.

46. Syed DN,Chamcheu JC,Adhami VM and Mukhtar H: Pomegranate extracts and cancer prevention: Molecular and cellular activities. Anticancer Agents Med Chem 13: 1149-1161, 2013.

This work is licensed under a Creative Commons Attribution-NonCommercial-NoDerivatives 4.0 International (CC BY-NC-ND 4.0) License. 